\title{
Fatores Ambientais e Parâmetros Genéticos para Características Produtivas e Reprodutivas em um Rebanho Nelore no Estado do Mato Grosso do Sul
}

\author{
Jânio Coelho da Silveira ${ }^{1}$, Concepta McManus ${ }^{1}$, Arthur dos Santos Mascioli ${ }^{1}$, Luiz Otávio \\ Campos da Silva ${ }^{2}$, Arley Coelho da Silveira ${ }^{3}$, José Américo Soares Garcia ${ }^{1}$, Helder Louvandini ${ }^{1}$
}

RESUMO - Objetivou-se no presente trabalho investigar os efeitos de alguns fatores ambientais e estimar componentes de (co)variâncias e parâmetros genéticos para as características produtivas de peso à desmama (PD) e aos 18 meses (P18) e características reprodutivas de perímetro escrotal aos 18 meses (PE18), intervalo de partos (IEP) e fertilidade real (FR), idade ao primeiro parto (IPP) e data de parto (DP) de fêmeas Nelore, em um rebanho do Estado de Mato Grosso do Sul. As observações, referentes aos fatores ambientais, foram analisadas pelo método dos quadrados mínimos, cujos modelos estatísticos incluíram os efeitos fixos de ano (AN) e mês de nascimento (MN), a interação AN*MN, sexo (S) do animal (exceto para P18, PE18 e IPP) ou do bezerro e ordem do parto. Verificaram-se efeitos significativos de todos os fatores estudados sobre PD, com os mais leves em agosto (179 kg) e os mais pesados em dezembro (156 kg). Os machos $(172 \mathrm{~kg}$ ) foram 7,4\% mais pesados que as fêmeas $(162 \mathrm{~kg})$. O P18 foi influenciado apenas pelo ano de nascimento. Não foi observada tendência, ao longo dos anos, para esta característica. Observou-se que somente a ordem do parto não influenciou o PE18 (média $281 \mathrm{~kg}$ ). Todos os efeitos foram significativos para IEP (média 465 dias), com exceção do sexo do bezerro, que não apresentou influência significativa. A FR foi influenciada por todos os efeitos, apresentando valor médio de $144 \mathrm{~kg}$. IPP (média 1.279 dias ou 41,93 meses) e DP sofreram influência dos efeitos fixos de mês e ano de nascimento, havendo também interação entre mês e ano de nascimento neste caso. Com base nas estimativas de herdabilidade, ambiente permanente, correlações genéticas e fenotípicas, calculadas por meio do programa MTDFREML, concluiu-se que as características estudadas podem responder à seleção direta, com exceção da FR e da IPP. As correlações genéticas entre as características produtivas estudadas foram favoráveis, indicando possibilidade na obtenção de progressos genéticos indiretos.

Palavras-chave: dia do parto, fertilidade real, idade ao primeiro parto, intervalo de partos, perímetro escrotal, pesos

\section{Study of Genetic and Environmental Factors on Production and Reproduction Traits in a Nellore Herd in Mato Grosso do Sul State}

\begin{abstract}
The objective of the present work was to investigate the effects of some genetic and environmental factors on production (weaning weight - WW; eighteen month weight - W18) and reproduction (scrotal perimeter at 18 months - SP18; calving interval - CI; age at first calving - AFC; True fertility - TF, calving date - CD) traits in a Nellore cattle herd in the Mato Grosso do Sul State, Brazil. The observations were analysed using the least squares methodology, and the statistical models included the fixed effects: year (YB) and month (MB) of calving, interaction YB*MB, sex (S) of animal (except for W18, SP18 and AFC) and calving number. Significant effects were found for all factors studied on WW, and animals born in August (179 kg) and December (156 kg) had highest and lowest WW respectively. The male calves $(174 \mathrm{~kg})$ were heavier at weaning than females $(162 \mathrm{~kg})$, a difference of $7.4 \%$ in favour of males. No tendency was observed in terms of mean values over the years for this trait. W18 was influenced only by birth year, with a mean value of $281.0 \mathrm{~kg}$. Only calving number did not influence Scrotal perimeter (SP18), which had a mean value of $26.8 \mathrm{~cm}$. The mean CI was 465 days, and all effects were significant, except for calf sex. TF was significantly influenced by all effects studied, and had a mean value of $144 \mathrm{~kg}$. Age at first calving was high (1.279 days or 41.93 months) and influenced by month and year of calving. In terms of calving date, month and year were significant effects, as was the interaction between these effects but sex and calving number did not affect this trait. Based on estimates of heritability, permanent environment, as well as on genetic and phenotypic correlations, estimated using MTDFREML, it was concluded that these traits should respond to direct selection, except for RF and AFP. Genetic correlations between production traits were favourable, indicating the possibility of genetic progress through indirect selection.
\end{abstract}

Key Words: age at first calving, calving date, calving interval, weight, scrotal perimeter, true fertility

\footnotetext{
${ }_{1}^{1}$ Faculdade de Agronomia e Medicina Veterinária, Universidade de Brasília, Cx P 04508, CEP: 70910-900, Brasília-DF (concepta@unb.br). ${ }_{2}^{2}$ Pesquisador da Embrapa Gado de Corte (CNPGC), Caixa Postal 154, CEP: 79002-970, Campo Grande-MS.

3 Departamento de Medicina Veterinária, Universidade Federal de Mato Grosso do Sul, Caixa Postal 649, Campo Grande-MS.
} 


\section{Introdução}

A bovinocultura de corte brasileira, com o maior rebanho comercial do mundo, constituída em sua maioria por raças zebuínas - com eficiente interação com o ambiente tropical - e explorada basicamente em sistema de produção a pasto, é um concorrente para outros países produtores de carne, pois vem passando por grandes transformações, em que se busca meios para elevar a produtividade e eficiência econômica e produtiva do setor, tornando-a uma atividade atraente e competitiva. Nesse contexto, a eficiência reprodutiva dos rebanhos é um dos fatores determinantes na eficiência total de produção e deve, portanto, ser considerada como critério de seleção em programas de melhoramento animal. Entretanto, para que o melhoramento das características de importância econômica seja efetivo, faz-se necessário conhecer os fatores ambientais ou as fontes de variação não-genéticas que atuam sobre estas características, interferindo no crescimento e desenvolvimento dos rebanhos de corte no Brasil.

Com a raça Nelore, existem vários estudos (Nájera et al., 1991; Souza et al., 1994) que identificaram fontes de variação não-genéticas (ano de nascimento, mês de nascimento, idade da vaca ou ano do parto) sobre as características produtivas e reprodutivas. Porém, é necessário que essas fontes sejam identificadas e estudadas no próprio ambiente, obtendo-se, posteriormente, fatores de correção.

Diante disso, objetivou-se, neste trabalho, estudar a influência de alguns fatores ambientais e estimar componentes de (co)variância e parâmetros genéticos para características produtivas (peso ajustado para 205 e 550 dias, perímetro escrotal ajustado para 550 dias) e reprodutivas (fertilidade real, idade ao primeiro parto, intervalo de partos e dias desde o início da estação de parição) em um rebanho Nelore no Estado do Mato Grosso do Sul.

\section{Material e Métodos}

Os dados utilizados referem-se a um rebanho de bovinos da raça Nelore, pertencentes à Fazenda Califórnia, localizada no município de Água Clara, na região nordeste do Estado do Mato Grosso do Sul. A propriedade está localizada à uma altitude variando de 550 a 600. O clima, de acordo com a classificação de Köppen, é tropical tipo AW, caracterizado por duas estações - uma seca (abril a setembro) e outra chuvosa (outubro a março) -, com precipitação média anual de $1.680 \mathrm{~mm}$. Em função da distribuição irregular das chuvas, a disponibilidade de forragens nesta região é irregular, com excedente em um período e escassez no outro.

A alimentação foi exclusivamente em regime de pasto, formado principalmente por Brachiaria decumbens (85\%) e Brachiaria brizantha (15\%), com suplementação mineral ad libitum, em cochos cobertos. O manejo do rebanho foi feito com cronograma pré-estabelecido de controle sanitário, não existindo um sistema eficiente de recursos de forragem para utilização no período de escassez.

A origem dos animais foi na década de 1970, quando se iniciou de maneira empírica o processo de seleção. Somente a partir de 1982 é que se deu início à coleta de dados de desempenho destes animais puros de origem (PO) e puros por cruza (LA), originando o banco de dados hoje existente e utilizado neste estudo. Os acasalamentos foram direcionados individualmente, considerando-se a genealogia dos animais, dando-se preferência aos acasalamentos exogâmicos, com o intuito de evitar os efeitos depressivos da consangüinidade e elevar a variabilidade genética do rebanho. Portanto, os acasalamentos foram direcionados de forma divergente ou compensatória, ou seja, procurou-se compensar a possível deficiência da fêmea em determinada característica com a utilização de um reprodutor com desempenho superior no referido caráter.

Existe estação de inseminação artificial definida, no período das águas (novembro a fevereiro), para a qual os reprodutores são escolhidos de acordo com a genealogia, desempenho individual ou da progênie e com sua classificação nos sumários de touros, de acordo com as DEPs (Diferença Esperada na Progênie) para os pesos às idades padronizadas aos $205 \mathrm{e}$ 550 dias e perímetro escrotal aos 550 dias. As fêmeas foram inseminadas artificialmente por até duas vezes durante a estação de monta, sendo manejadas a partir do terceiro cio em sistema de monta natural.

Foi realizado descarte dos animais defeituosos na ocasião da desmama, das fêmeas no momento de sua entrada na estação de inseminação e das fêmeas em reprodução, conforme o baixo desempenho reprodutivo.

Foram estudadas as características de peso à desmama (PD), corrigido para 205 dias, peso aos 18 meses (P18), corrigido para 550 dias, perímetro escrotal aos 550 dias (PE18), fertilidade real (FR), intervalo de partos (IEP), idade ao primeiro parto

\section{R. Bras. Zootec., v.33, n.6, p.1432-1444, 2004}


(IPP) e data do parto (DP) de, respectivamente, 2952, $551549,1.749,3.069,1.080$ e 4.073 animais nascidos de 1982 a 1999. A FR foi estimada de acordo com a fórmula descrita por Lobo et al. (1994), citada e utilizada por Campello et al. (1999):

$$
\mathrm{FR}=\frac{\mathrm{PD} \times 365 \text { dias }}{\mathrm{IEP}}
$$

Os dados referentes aos fatores ambientais foram analisados pelo método dos quadrados mínimos, utilizando-se o procedimento GLM (SAS, 1999), sendo que os modelos estatísticos incluíram os efeitos fixos de ano e mês de nascimento ou do parto, ordem do parto e sexo do animal.

Para a estimativa dos componentes de (co)variância utilizados na estimativa de herdabilidade e das correlações genéticas, fenotípicas e ambientes, utilizou-se o método de máxima verossimilhança restrita livre de derivadas sob modelo animal. Foram realizados os cálculos de herdabilidade, ambiente permanente, correlações genética e fenotípica adotando-se o programa MTDFREML (Multiple Trait Derivate Free Restricted Maximum Likelihood), segundo Boldman et al. (1995), ajustando-se a equação para peso à desmama:

$$
Y=X b+Z_{1} a+Z_{2} m+Z_{3} p e+e
$$

em que $Y$ é um vetor (Nx1) de observação do animal; $b$, o vetor de efeitos fixos (sexo, mês, ano de nascimento e ordem da parição e características de reprodução) no modelo, associado com o matriz de incidência $X$; $a$, o vetor dos efeitos genéticos diretos, associado com a matriz de incidência $Z_{1} ; m$, o vetor dos efeitos genéticos maternos, associado com a matriz de incidência $Z_{2}$; pe, o vetor dos efeitos de ambiente materno permanente, associado com a matriz de incidência $Z_{3}$; e $e$, o vetor de resíduos aleatórios. As características pós-desmama não incluíram os efeitos genéticos maternos ou o ambiente materno permanente. Os efeitos fixos utilizados para cada característica foram ano e mês de nascimento do bezerro, além de sexo e ordem de parição/nascimento, quando aplicável.

Para as características de IPP, PE18 e P18, os efeitos materno e de ambiente permanente não foram colocados no modelo. Para IPP, ordem do parto e sexo do bezerro não foram incluídos como efeitos fixos no modelo. Para as características DP, FR e IEP, o efeito materno não foi incluído no modelo.

\section{Resultados e Discussão}

\section{Fatores ambientes}

O resumo das análises de variância para as características estudadas encontra-se na Tabela 1. Os coeficientes de determinação foram, em geral mais altos da literatura (Ribeiro et al., 2001; Rosa et al., 2001) e os coeficientes de variação mais baixos (Ribeiro et al., 2001; Siqueira et al. (2003).

Verificou-se efeito significativo $(\mathrm{P}<0,01)$ de todas as fontes de variação estudadas sobre peso à desmama (PD). Este resultado é bastante semelhante aos citados para esta característica (Silva et al., 2000; Oliveira et al., 1995). Sabe-se que o peso à desmama é pouco influenciado pela ação gênica aditiva e muito pela ação gênica não-aditiva (Giannoni \& Giannoni, 1989) e que os bezerros nascidos na primavera têm maiores pesos à desmama (Pereira, 1999). O mês de nascimento mais favorável para $\mathrm{PD}$ foi agosto (Tabela 2 ), que caracteriza, portanto, o final da época fria do ano e de baixa precipitação pluviométrica, sendo mais favorável à criação de bezerros, em razão da menor incidência de pragas e doenças. As vacas paridas no final da época seca têm boa disponibilidade de forragem para a maior parte da sua lactação, o que também favorece o crescimento dos bezerros (McManus et al., 2002; Mascioli et al., 1996).

Observaram-se variações nas médias de ano para ano e nenhuma tendência de aumento ou redução. Essas variações podem ser atribuídas às oscilações na disponibilidade e qualidade das forrageiras, em conseqüência das condições climáticas e dos manejos nutricional, sanitário e reprodutivo aplicado aos animais em cada ano, especialmente às vacas. $\mathrm{O}$ ambiente proporcionado às vacas, dentro de cada ano, também tem influência indireta sobre o crescimento pré-desmama. Efeitos de ano de nascimento foram observados por Milagres et al. (1985), Nobre et al. (1985), Nájera et al. (1991) e Mascioli et al. (1996). O efeito da interação ano*mês de nascimento encontrado neste estudo reflete as argumentações acima, porém evidencia a tendência dos maiores pesos à desmama nos nascimentos de agosto e setembro, seguido por decréscimo nos meses subseqüentes.

O sexo do animal também apresentou efeito significativo $(\mathrm{P}<0,01)$ sobre $\mathrm{PD}$ e as médias mínimas quadrados segundo o sexo podem ser verificados na Tabela 2. O peso médio dos machos foi de $174 \mathrm{~kg}$ e o das fêmeas foi de $162 \mathrm{~kg}$, observando-se diferença em favor dos machos de 7,4\% - semelhante aos

\section{R. Bras. Zootec., v.33, n.6, p.1432-1444, 2004}


Tabela 1 - Resumo da análise de variância do peso à desmama (PD), 550 dias (P18), perímetro escrotal aos 550 dias (PE18), fertilidade real (FR), intervalo de parto (IEP), idade ao primeiro parto (IPP) e data do parto (DP) em um rebanho Nelore

Table 1 - Summary of variance analysis for weaning weight (WW), 18 month weight (W18), scrotal perimeter at 18 months (SP18), calving interval (CI), age at first calving (AFC), true fertility (TF) and calving date (CD) in a Nellore herd

\begin{tabular}{|c|c|c|c|c|c|c|c|c|}
\hline \multirow[b]{2}{*}{ Fonte de variação } & \multirow[b]{2}{*}{ GL } & \multicolumn{7}{|c|}{$\begin{array}{l}\text { Nível de significância } \\
\text { Significance level }\end{array}$} \\
\hline & & PD & P18 & PE18 & IEP & FR & IPP & DP \\
\hline Source of variation & $D F$ & $W W$ & $W 18$ & $S P 18$ & $C I$ & $T F$ & $A F C$ & $C D$ \\
\hline $\operatorname{Sexo}(S)$ & 1 & $* * *$ & - & - & $\mathrm{ns}$ & $*$ & - & $*$ \\
\hline \multicolumn{9}{|l|}{ Sex } \\
\hline Mês nasc. (MN) & 4 & $* * *$ & $* * *$ & $*$ & $*$ & $*$ & $* *$ & $* *$ \\
\hline \multicolumn{9}{|c|}{ Month of calving (MC) } \\
\hline Ano nasc. (AN) & $6(5)^{\mathrm{a}}$ & $*$ & $*$ & $* *$ & $* *$ & $* *$ & $* *$ & $*$ \\
\hline $\begin{array}{l}\text { Year of calving }(Y C) \\
\mathrm{AN}^{*} \mathrm{MN} M C^{*} Y C\end{array}$ & $24^{b}$ & $*$ & $\mathrm{~ns}$ & $* *$ & $*$ & $* *$ & $\mathrm{~ns}$ & $*$ \\
\hline $\mathrm{N}^{\mathrm{o}}$ parto $(\mathrm{NP})$ & $7^{c}$ & $*$ & $\mathrm{~ns}$ & $\mathrm{~ns}$ & $*$ & $* 8$ & - & $\mathrm{ns}$ \\
\hline \multicolumn{9}{|l|}{ Calving number } \\
\hline Touro (T) & 61 & $* * *$ & $* *$ & $* *$ & - & $* *$ & - & - \\
\hline $\begin{array}{l}\text { Bull } \\
\mathrm{R}^{2}(\%)\end{array}$ & & 64 & 63 & 71 & 37 & 55 & 43 & 94 \\
\hline $\mathrm{CV}$ & & 10,62 & 11,24 & 13,02 & 21,33 & 16,35 & 12,10 & 10,50 \\
\hline
\end{tabular}

a Graus de liberdade para PD entre parênteses.

b Graus de liberdade para P18, PE18, IEP, FR e IPP igual 3, 3, 5, 5 e 7, respectivamente.

c Graus de liberdade para P18, PE18, IDP, FR e IPP igual 12, 12, 20, 20 e 28, respectivamente.

d Graus de liberdade para erro igual 524, 522, 3011, 1711, 1040 e 4005 para PD, P18, PE18, IDP, FR, IPP e DP, respectivamente.

${ }^{* * *}=\mathrm{P}<0,001 ;{ }^{* *} \mathrm{P}<0,01,{ }^{*} \mathrm{P}<0,05, \mathrm{CV}-$ coeficiente de variação.

a Degrees of freedom for WW within parenthesis.

${ }^{b}$ Degrees of freedom for W18, SP18, CI, TF and AFC equal to 3,3, 5, 5 e 7, respectively.

${ }^{c}$ Degrees of freedom for W18, SP18, CI, TF and AFC equal to 12, 12, 20, 20 and 28, respectively.

${ }^{d}$ Degrees of freedom of the error equal to 524, 522, 3011, 1711, 1040 and 4005 for W18, SP18, CI, TF, AFC and CD, respectively.

${ }^{* * *} P<.001 ;{ }^{* *} P<.01,{ }^{*} P<.05, \quad C V-$ coefficient of variation.

Tabela 2 - Médias de quadrados mínimos, de acordo com o mês de nascimento (MN), sexo do animal ou do bezerro (S) e ordem do parto (NP)

Table 2 - Least square means per month of calving (MC), calf sex (S) and calving number (CN)

\begin{tabular}{|c|c|c|c|c|c|c|c|}
\hline $\begin{array}{l}\text { Efeitos } \\
\text { Effects }\end{array}$ & $\begin{array}{l}\mathrm{PD}(\mathrm{kg}) \\
W W(k g)\end{array}$ & $\begin{array}{l}\mathrm{P} 18(\mathrm{~kg}) \\
W 18(\mathrm{~kg})\end{array}$ & $\begin{array}{l}\text { PE18 }(\mathrm{cm}) \\
S P 18(\mathrm{~cm})\end{array}$ & $\begin{array}{l}\text { IEP (dias) } \\
C I \text { (days) }\end{array}$ & $\begin{array}{l}\mathrm{FR}(\mathrm{kg}) \\
T F(k g)\end{array}$ & $\begin{array}{l}\text { IPP (dias) } \\
A F C \text { (days) }\end{array}$ & $\begin{array}{l}\mathrm{DP} \text { (dias) } \\
C D \text { (days) }\end{array}$ \\
\hline$\overline{\mathrm{MN}}$ & \multicolumn{7}{|c|}{ Médias $\pm \mathrm{EP}^{1}$} \\
\hline$M C$ & & & & Means $\pm S E^{l}$ & & & \\
\hline Agosto (August) & $179 \pm 1$ & $275 \pm 7$ & $28 \pm 0,5$ & $421 \pm 6$ & $160 \pm 2$ & $1333 \pm 16$ & $200 \pm 0,5$ \\
\hline Setembro (September) & $177 \pm 1$ & $287 \pm 5$ & $28 \pm 0,5$ & $437 \pm 5$ & $156 \pm 2$ & $1314 \pm 12$ & $46 \pm 0,5$ \\
\hline Outubro (October) & $168 \pm 1$ & $291 \pm 4$ & $27 \pm 0,5$ & $440 \pm 5$ & $145 \pm 2$ & $1269 \pm 13$ & $76 \pm 0,4$ \\
\hline Novembro (November) & $162 \pm 1$ & $283 \pm 4$ & $26 \pm 0,5$ & $450 \pm 6$ & $145 \pm 2$ & $1204 \pm 15$ & $107 \pm 0,4$ \\
\hline Dezembro (December) & $156 \pm 1$ & $292 \pm 5$ & $26 \pm 0,5$ & $483 \pm 4$ & $134 \pm 2$ & $1243 \pm 11$ & $136 \pm 0,4$ \\
\hline Janeiro (January) & - & - & - & - & - & - & $167 \pm 0,4$ \\
\hline \multicolumn{8}{|l|}{ S } \\
\hline Machos (Males) & $174 \pm 1$ & - & - & $448 \pm 3$ & $153 \pm 1$ & - & $122 \pm 0,2$ \\
\hline Fêmeas (Females) & $162 \pm 1$ & - & - & $445 \pm 3$ & $144 \pm 1$ & - & $122 \pm 0,2$ \\
\hline \multicolumn{8}{|l|}{$\mathrm{NP}(C N)$} \\
\hline 1 & $162 \pm 1$ & $282 \pm 4$ & $27 \pm 0,5$ & $517 \pm 4$ & $125 \pm 2$ & - & $122 \pm 0,3$ \\
\hline 2 & $169 \pm 1$ & $282 \pm 4$ & $27 \pm 0,5$ & $453 \pm 4$ & $145 \pm 2$ & - & $122 \pm 0,4$ \\
\hline 3 & $171 \pm 1$ & $287 \pm 4$ & $27 \pm 0,5$ & $433 \pm 5$ & $152 \pm 2$ & - & $122 \pm 0,4$ \\
\hline 4 & $171 \pm 1$ & $291 \pm 4$ & $27 \pm 0,5$ & $441 \pm 6$ & $152 \pm 2$ & - & $122 \pm 0,4$ \\
\hline 5 & $171 \pm 1$ & $290 \pm 5$ & $27 \pm 0,5$ & $434 \pm 6$ & $154 \pm 2$ & - & $122 \pm 0,5$ \\
\hline 6 & $169 \pm 1$ & $292 \pm 5$ & $27 \pm 0,5$ & $427 \pm 8$ & $155 \pm 2$ & - & $122 \pm 0,6$ \\
\hline 7 & $169 \pm 1$ & $278 \pm 8$ & $26 \pm 0,5$ & $423 \pm 10$ & $155 \pm 3$ & - & $121 \pm 0,7$ \\
\hline 8 & $165 \pm 1$ & $280 \pm 9$ & $27 \pm 0,5$ & $440 \pm 11$ & $147 \pm 3$ & - & $122 \pm 0,7$ \\
\hline
\end{tabular}

1 PD, peso à desmama; P18, peso aos 550 dias de idade; PE18, perímetro escrotal aos 550 dias de idade; IEP, intervalo de partos; FR, fertilidade real; IPP, idade ao primeiro parto; DP, data do parto, EP = erro-padrão.

1 Weaning weight (WW), 18 month weight (W18), scrotal perimeter at 18 months (SP18), calving interval (CI), age at first calving (AFC), true fertility (TF) and calving date $(C D), S E=$ standard error .

R. Bras. Zootec., v.33, n.6, p.1432-1444, 2004 
valores de 6,4 e 7,0\% encontrados por Arias \& Slobodzian (1998) e Mascioli et al. (1996). Mariante \& Zancaner (1985) observaram diferença média de $22,5 \mathrm{~kg}$, representando $12 \%$ em favor dos machos, também na raça Nelore.

Selecionando-se apenas para maiores pesos à desmama, pode-se estar também selecionando aqueles animais de maior porte e que, à idade adulta, terão maior peso e, no caso dos machos, poderão apresentar acabamento de carcaça tardiamente. No caso de fêmeas, pode-se também observar antagonismos no sentido de diminuir a eficiência reprodutiva, uma vez que poderá ocorrer aumento da idade ao primeiro parto em função de maior período da fase de crescimento, retardando o início dos eventos reprodutivos (para rebanhos sob baixo nível nutricional, como neste caso), além do maior peso à idade adulta, que exigirá maiores requerimentos de mantença, resultando em maiores intervalos de partos e, conseqüentemente, menor fertilidade real. Segundo Rosa \& Lobo (1998), ao se procurar o crescimento da produção em valor absoluto geralmente, aumenta, como resposta correlacionada, o tamanho à idade adulta, com consequiências negativas sobre o custo de mantença, uma vez que, quanto maior o tamanho, maior será o consumo de alimentos. Outras implicações neste sentido podem são a diminuição da taxa de maturidade e problemas relacionados à harmonia, à funcionalidade e ao equilíbrio do organismo animal. Além disso, os bezerros maiores não-selecionados para maior peso à desmama também podem sofrer problemas de distocia.

A ordem do parto também afetou $(\mathrm{P}<0,05)$ o peso à desmama do bezerro, corroborando os resultados encontrados por vários autores (Arias \& Slobodzian, 1998; Mariante \& Zancaner, 1985; Souza et al., 1994; Mascioli et al., 1996). Houve aumento no peso à desmama do primeiro ao terceiro parto (Tabela 2), com posterior estabilização até o quinto, quando ocorreu redução a partir do sexto parto. Assim, entende-se que as vacas mais novas (primíparas) tendem a ter menor produção de leite que as mais velhas (multíparas), até certa idade.

Para a característica peso aos 18 meses, utilizaram-se apenas as informações dos machos, que totalizaram 551 animais. Não houve influência significativa dos efeitos estudados, exceto do ano de nascimento. Segundo Mascioli et al. (1996), o efeito de ano tem influência direta sobre o animal, podendo também refletir mudanças na média do valor genético do rebanho, como consequiência de um programa de seleção ou da introdução de touros e, ou, de vacas geneticamente superiores. Esse efeito de ano pode ser atribuído às diferenças no tamanho das amostras de ano para ano (Oliveira, 1979).

Para os bezerros nascidos no início da estação de nascimento (agosto e setembro), que apresentaram maiores pesos à desmama, esperar-se-ia que alcançassem apresentassem maiores pesos ao sobreano (pleiotropia). Isto não ocorreu no rebanho analisado, o que poderia ser explicado pelo fato de que a maioria dos bezerros foi desmamada no início do período de menor disponibilidade de pastagens, acarretando baixo nível nutricional nesta fase em que o animal passaria a viver sem o ambiente maternal, acarretando pouco ou nenhum ganho de peso no período pós-desmama. Os bezerros nascidos no final da estação (dezembro e janeiro), desmamados em julho/ agosto com menor peso, passaram maior parte da fase crítica de escassez de forragem sob os cuidados da mãe e, ao final do período de adversidade climática, iniciaram a fase pós-desmama no período de maior disponibilidade de forragem, com ganhos de peso que permitiriam uma compensação para o menor peso à desmama.

A característica PE18, referente a 549 animais, apresentou valor médio de $26,4 \mathrm{~cm}$, ligeiramente inferior ao relatado por Gressler (1998), porém similar aos valores encontrados na literatura (Bergmann et al., 1996). Com exceção da ordem do parto, todos os efeitos influenciaram significativamente $(\mathrm{P}<0,001)$ esta característica, de modo semelhante ao achados de Neely et al. (1982) e Smith et al. (1989). O que poderia explicar esse resultado, em parte, seriam as mesmas condições que atuaram no peso à desmama, citadas anteriormente.

Segundo Bergmann (1999), a seleção para aumento do perímetro escrotal economicamente não traz benefícios diretos. Busca-se, contudo, com esta seleção, animais mais precoces sexualmente e mais férteis, com maior quantidade e qualidade espermática e maior capacidade de serviço. Existem evidências de que o perímetro escrotal esteja correlacionado geneticamente com a idade à puberdade nos machos e nas fêmeas, com a fertilidade das fêmeas aparentadas a estes machos (Gressler, 1998) e, ainda, com características físicas e morfológicas do sêmen.

Foram consideradas 3.069 observações para a característica IEP, sendo encontrado valor médio de 465 dias, inferior ao encontrado por McManus et al.

R. Bras. Zootec., v.33, n.6, p.1432-1444, 2004 
(2002) (483 dias), porém similar ao encontrado na literatura (Nájera, 1990). Se for considerado o IEP em base anual, que seria uma meta desejável para as condições deste trabalho, constata-se que ainda deverá ser reduzido em, aproximadamente, 21,5\%.

Para as condições deste trabalho, as análises demonstram que os efeitos de ano e mês de nascimento do bezerro constituem importante fonte de variação para esta característica. Ao analisar o período de 1989 a 1998, verifica-se que o ano de 1994 apresentou o maior valor para IEP, que poderia ser explicado pela má distribuição de chuvas durante o ano, contribuindo para a ocorrência de longo período de déficit hídrico, escassez de forragem e consequiente prejuízo para a recuperação do estresse do parto pelas matrizes. Resultados semelhantes foram obtidos por Miranda et al. (1982), em rebanho Nelore, e por Pädua et al. (1994), em animais da raça Canchim.

A ordem do parto teve efeito significativo ( $\mathrm{P}<0,001)$, ocorrência esperada, pois, segundo Pereira (1999), existem indicações de que as primíparas apresentam valores de IEP ligeiramente mais elevados que as multíparas, indicando que principalmente aquela categoria tem seu desempenho afetado pelo meio ambiente (Tabela 2) e apresenta o maior intervalo de partos, ressaltando-se que as matrizes de idade mais elevada também apresentam IEP mais elevado, o que está de acordo com os dados encontrados na literatura. Não houve efeito do sexo do bezerro sobre o IEP, fato também verificado por Cardellino \& Pons (1987) e Pädua et al. (1994).

Segundo Bergmann (1993), a utilização do IEP pode ser considerada tendenciosa, pois ocorre apenas em animais que tiveram pelo menos dois partos durante sua vida reprodutiva, além de que as vacas que parem cedo na estação de nascimento são forçadas a um longo período pós-parto antes da próxima estação de monta e consequiente IEP maior que 365 dias. Por outro lado, as matrizes que parem tardiamente terão um período mais curto entre o parto e o início da estação de monta e, conseqüentemente, apresentarão menor IEP.

Bourdon \& Brinks (1983) observaram que curtos intervalos de partos são precedidos por intervalos mais longos, fato também observado neste trabalho. As vacas que pariram nos meses de agosto e setembro apresentaram intervalos de partos superiores àquelas que pariram nos meses de outubro e novembro. Aquelas que pariram em agosto estão aptas a conceber a partir de meados de setembro, porém devem aguardar o início da estação de inseminação (novembro), o que poderá provocar aumento do IEP. Os partos ocorridos em dezembro ocasionaram os maiores intervalos de partos, provavelmente em função de as vacas necessitarem se restabelecer do estresse pós-parto já no final da estação de inseminação (meados de fevereiro), diminuindo o tempo de exposição do animal ao manejo de inseminação, além de se permitir a aplicação de uma única dose de sêmen antes do encerramento da estação de inseminação, determinando taxa de concepção inferior à observada para aqueles animais que pariram em fases iniciais da estação de nascimento.

Para a fertilidade real (FR), foram analisadas informações de 1.749 matrizes, que apresentaram valor médio de $144 \mathrm{~kg}$. A fertilidade real foi influenciada significativamente $(p<0,01)$ pelos efeitos fixos de ano e mês de nascimento, pela interação $\mathrm{AN}^{*} \mathrm{MN}$ e pela ordem de parto.

McManus et al. (2002) encontraram valores inferiores à estes $-113,9 \mathrm{~kg}$ para vacas com bezerros e $111,85 \mathrm{~kg}$ para vacas com bezerras. Todavia, Campelo et al. (1999) obtiveram valor médio de $185 \mathrm{~kg}$. Ainda assim, nota-se alta variabilidade fenotípica nos resultados, indicando que a característica é complexa, pois envolve os eventos reprodutivos, a habilidade materna e a expressão dos genes de crescimento em uma única característica.

A fertilidade real sofre influência direta do intervalo de partos em uma relação inversa e este, por sua vez, entre outros fatores, é dependente do período da estação de inseminação (Bourdon \& Brinks, 1983; Bergmann, 1993). Por outro lado, estes resultados poderiam indicar que o IDP atua sobre a FR em intensidade menor que o ganho de peso pré desmama, fator que afeta diretamente a FR. As vacas apresentaram valores decrescentes de FR a partir dos nascimentos no mês de agosto, mês com menor disponibilidade de forragem para a mãe, implicando em menor produção de leite para o bezerro. A exigência por parte do bezerro também é menor, em função de seu menor peso, e, à medida que o bezerro cresce, aumenta sua exigência em consumo. Paralelamente, há aumento da produção de leite da mãe, em função das melhorias no ambiente (clima), que resulta em maior disponibilidade de forragem para a mãe e para o bezerro até o final do período das águas, quando ocorre a desmama. Para os bezerros nascidos no final da estação, ocorre o inverso: há elevada produção de leite e oferta de forragem de boa qualidade em

\section{R. Bras. Zootec., v.33, n.6, p.1432-1444, 2004}


um momento de menor exigência pelo bezerro e, à medida que aumenta, suas exigência, diminui a oferta de forragem, proporcionando menores pesos à desmama.

Igualmente ao IEP, o ano e mês de nascimento e a sua interação também foram fontes de variação sobre a FR, resultado semelhante aos observados por McManus et al. (2002). Essas características seriam pouco influenciadas por genes aditivos e o melhoramento delas dependeria, fundamentalmente, de mudanças na qualidade de alimentação, de cuidados profilático-sanitários, do manejo reprodutivo etc. A pequena variabilidade genética aditiva seria explicada pela seleção natural que teria atuado desde a origem das diferentes raças. Este argumento é a base de sustentação de que mudanças ambientes seriam determinantes no melhoramento da eficiência reprodutiva, conforme sugerem pesquisadores norte-americanos (Hansen et al., 1983). Para as condições deste trabalho, observou-se que o ano que apresentou a menor fertilidade real $(1994,133 \mathrm{~kg})$ foi justamente aquele em que houve a pior distribuição de chuvas, impondo baixo nível nutricional ao rebanho e afetando diretamente o desempenho reprodutivo das vacas.

Os resultados registrados neste trabalho, para o efeito da ordem do parto sobre a FR, apresentam valores similares aos observados por Campelo et al. (1999), que apontam melhoria na FR até a quarta parição. Outro aspecto observado foi a fertilidade real relacionada ao $8 \underline{0}, 9 \underline{0}$ e $10^{\circ}$ partos não apresentar queda substancial em seus valores, o que era esperado, uma vez que as vacas apresentaram idades bastante elevadas das vacas por ocasião destes partos $( \pm 12$ anos), o que permite inferir que a seleção para eficiência reprodutiva aplicada no transcorrer dos anos fez com que apenas as vacas de alto desempenho conseguissem permanecer no rebanho, visto que poucos animais tiveram este número de partos.

O sexo do bezerro também foi uma fonte de variação que influenciou a fertilidade real, pois as vacas que amamentavam fêmeas apresentaram fertilidade real de $144 \mathrm{~kg}$, enquanto as que amamentavam machos, de $153 \mathrm{~kg}$, ou seja, a FR das vacas que amamentavam machos foi $6,5 \%$ maior que a das que amamentavam fêmeas.

Observou-se também efeito, de menor intensidade, do intervalo de partos na determinação da FR, quando comparado com o crescimento e ganho pré-desmama, pois, segundo Gressler (1998), vacas primíparas amamentando bezerros machos apresen- tam IEP superiores aos daquelas que amamentam fêmeas, o que acarretaria menores valores para a FR. Porém, como a FR também depende do crescimento e ganho de peso pré-desmama do bezerro e, como os machos são mais pesados que as fêmeas, ocorre inversão, prevalecendo FR de maior magnitude para as vacas que amamentam bezerros machos, mesmo que estas apresentem maiores IEP.

Para a variável idade ao primeiro parto, foram analisadas observações de 1.080 animais, com média de 1.279 dias de idade, correspondendo a 41,93, e coeficiente de variação de $13 \%$. O valor obtido é semelhante ao relatado por Nájera (1990), que descreve registros de vários estudos com animais da raça Nelore no Brasil, porém esta semelhança não foi observada em relação ao desvio-padrão, e os resultados aqui relatados foram superiores à média. Vários trabalhos têm demonstrado a vantagem em se iniciar mais cedo a vida reprodutiva das novilhas. Teixeira (1997), ao simular três sistemas de produção para novilhas Nelore, verificou que a antecipação do primeiro parto para os 27-30 meses de idade acarretaria, entre outras vantagens, em acréscimo na receita bruta da atividade pecuária.

Neste estudo, houve ef eito significativo $(\mathrm{P}<0,001)$ do mês de nascimento e ano de nascimento sobre IPP, indicando que são importantes fontes de variação. Analisando-se o efeito de mês de nascimento, verifica-se tendência de decréscimo na idade ao primeiro parto a partir do mês de agosto (maior IPP, 1.333 dias), sendo novembro o mês mais favorável para diminuição da IPP (Tabela 2 ). O que poderia explicar, em parte, este resultado, seria o deliberado atraso pelo criador do início da estação de inseminação para as novilhas nascidas no início da estação de nascimento e que, mesmo aptas à reprodução, acabam penalizadas no sentido de aguardarem as demais para o início da fase de reprodução, fato também observado por Gressler (1998). Conforme Pereira (1999), o peso à desmama tende a aumentar a partir dos nascimentos do mês de setembro e, neste estudo, observou-se o aumento a partir do mês de agosto, portanto, esperar-se-ia que estes animais atingissem mais precocemente o início da atividade reprodutiva, fato que não ocorreu.

Considerando que a bovinocultura brasileira é baseada na exploração a pasto e sabendo-se que as variações climáticas entre anos sobre a qualidade e disponibilidade de forragens, este estudo confirma o efeito do ano de nascimento como fonte de variação

\section{R. Bras. Zootec., v.33, n.6, p.1432-1444, 2004}


( $\mathrm{P}<0,001$ ), sobre o início da puberdade das fêmeas e, conseqüentemente, a idade ao primeiro parto, evidenciando ser a IPP dependente das condições nutricionais às quais é submetido o rebanho. Porém, no decorrer do período analisado, houve certa estabilidade para a característica, com o ano de 1999 tendo pequena elevação na idade ao primeiro parto (Tabela 2). Visto que este ano apresentou o menor índice pluviométrico registrado em 18 anos (29,9\% abaixo da média), tal ocorrência explicaria, em parte, o atraso na idade ao primeiro parto neste ano, em decorrência da menor disponibilidade de forragens durante a fase de crescimento das novilhas.

De acordo com Notter (1995), a utilização da IPP como critério de seleção não é adequada em países tropicais, pois esta característica já ocorre tardiamente, além de ser, geralmente, atrasada pela maioria dos produtores. Outro fator é o permanente nível nutricional baixo para o rebanho analisado, ou seja, o manejo das pastagens sempre tende a uma taxa de lotação superior à sua capacidade de suporte, impedindo melhores desempenhos para os animais de potencial genético superior.

A data do parto foi significativamente $(\mathrm{P}<0,001)$ influenciada pelo ano e mês de nascimento e pela interação entre esses efeitos (Tabela 1), sendo a DP influenciada de maneira diversa, ao longo dos anos; contudo, não houve efeito significativo do sexo do bezerro e da ordem de parto da vaca. Segundo Morris \& Cullen (1988), em rebanhos de estação de monta restrita, a data do parto não seria uma característica importante, em função da correlação negativa com o intervalo de partos, indicando que as vacas que parem cedo necessitam aguardar maior tempo para o início da próxima estação de monta que aquelas que parem tardiamente. Gressler (1998) sugere que a seleção para antecipação da data do parto resulta em aumento na taxa de parição e em antecipação da data do próximo parto, em estações de monta de curta duração (90 dias). Segundo Rege \& Famula (1993), a data do primeiro parto afeta as datas dos partos subseqüentes. De acordo com Bourdon \& Brinks (1983), uma vantagem da data do parto como medida da eficiência reprodutiva seria sua associação com a idade à desmama, ou seja, os bezerros que nascem mais cedo seriam mais velhos e pesariam mais à desmama quando esta fosse realizada em data fixa.

\section{Parâmetros genéticos}

As estimativas dos componentes de variância genética $\left(\hat{\sigma}_{\mathrm{a}}^{2}\right)$ e materna $\left(\hat{\sigma}_{\mathrm{m}}^{2}\right)$, ambiente permanente $\left(\hat{\sigma}^{2}{ }_{p e}\right)$, (co)variância e parâmetros genéticos de PD, P18 e PE18, IPP, DP, IEP e FR, em animais da raça Nelore de um rebanho no Mato Grosso do Sul, estão descritos na Tabela 3.

As correlações genéticas entre as características de crescimento observadas neste trabalho referentes às características produtivas entre PD e P18, PD e PE18 e P18 e PE18 encontram-se na Tabela 4.

$\mathrm{O}$ peso à desmama (PD) apresentou estimativa de herdabilidade do efeito genético aditivo direto de 0,17 (Tabela 3), semelhante à obtida por Ribeiro et al. (2001), mas inferior à relatada por Rosa (2001) e Siqueira et al. (2003), permitindo inferir que o rebanho está sujeito a modificações pela seleção e, apesar da baixa magnitude, é possível obter ganhos genéticos para a característica.

A variação resultante de efeitos do ambiente (alimentação, manejo etc) pode, em alguns casos, ser decomposta em fatores de ambiente permanente e de ambiente temporário (Trovo \& Razook, 1996). Os efeitos de ambiente permanente ocorrem em determinado período da vida do animal, afetando seu desempenho pelo resto da sua vida produtiva no rebanho. Já os efeitos de ambiente temporário são decorrentes de fatores eventuais, associados ao meio ambiente e afetam temporariamente o desempenho dos animais.

Ribeiro et al. (2001) e Garnero et al. (1998) observaram valores altos e negativos para as correlações genéticas entre os efeitos diretos e maternos dos pesos à desmama, distintos dos encontrados neste estudo $(0,27)$ (Tabela 4). Segundo Perotto (2000), o crescimento na fase compreendida entre o nascimento e à desmama de um bezerro é influenciado não apenas pelo seu genótipo, como também pelo ambiente proporcionado pela mãe. O ambiente materno é estritamente ambiental em relação ao bezerro, mas diferenças entre mães para este efeito são determinadas por causas genéticas e por fatores do meio. Como o bezerro recebe a metade de seus genes da mãe e a outra metade do touro, o resultado é que a mãe influencia o crescimento de seus filhos de duas maneiras: uma por meio dos genes que transmite e outra pelo ambiente materno que proporciona. 
Tabela 3 -Estimativas dos componentes de variância genética direta $\left(\hat{\sigma}^{2}{ }_{a}\right)$ e materna $\left(\hat{\sigma}^{2}\right.$ m), ambiente permanente $\left(\hat{\sigma}^{2}\right.$ pe $)$, covariância e parâmetros genéticos de peso à desmama (PD), aos 18 (P18) e perímetro escrotal aos 18 meses de idade (PE18), fertilidade real (FR), intervalo de partos (IEP), idade ao primeiro parto (IPP) e data do parto (DP) em animais da raça Nelore de um rebanho no MS

Table 3 - Estimates of direct $\left(\hat{\sigma}^{2}{ }_{A}\right)$, and maternal $\left(\hat{\sigma}^{2}{ }_{M}\right)$ (co)variance components and genetic parameters for weaning weight (WW), weight at 18 months of age (W18), scrotal perimeter at 18 months (SP18), true fertility (TF), calving interval (CI), age at first calving (AFC) and calving date (CD) for a Nellore herd in MS

Estimativas*

Estimates

\begin{tabular}{|c|c|c|c|c|c|c|c|}
\hline & $\mathrm{PD}(W W)$ & $\mathrm{P} 18(W 18)$ & PE18 (SP18) & $\mathrm{FR}(T F)$ & $\operatorname{IEP}(C I)$ & $\operatorname{IPP}(A F C)$ & $\mathrm{DP}(C D)$ \\
\hline$\hat{\sigma}^{2} \mathrm{a}$ & 63,191 & 389,428 & 2,572 & 398,194 & 1134,128 & 17,806 & 26,96 \\
\hline$\hat{\sigma}^{2} \mathrm{~m}$ & 34,48 & - & - & - & - & - & - \\
\hline$\hat{\sigma}^{2}$ pe & 48,41 & - & - & 2322,800 & 81,009 & - & 4,90 \\
\hline$\hat{\sigma}_{\mathrm{e}}^{2}$ & 260,138 & 908,655 & 4,022 & 3915,574 & 1386,156 & 338,314 & 42,547 \\
\hline$c^{2}$ & 0,13 & - & - & 0,35 & 0,03 & - & 0,06 \\
\hline $\mathrm{h}^{2} \mathrm{~m}$ & 0,08 & - & - & - & - & - & - \\
\hline $\mathrm{h}^{2{ }^{111}}$ & 0,17 & 0,30 & 0,39 & 0,06 & 0,42 & 0,05 & 0,33 \\
\hline
\end{tabular}

${ }^{*}$ A estimativa da covariância entre efeitos diretos e maternos para PD foi de 13,19 .

* The estimate covariance between direct and maternal effects for WW was13.19.

A estimativa de herdabilidade do P18 para este rebanho apresentou valor de 0,30 , ligeiramente superior às estimativas de outros autores na raça Nelore (Eler et al., 1994 e 1995; Magnabosco et al., 1996; Mercadante et al., 1997), que variaram de 0,16 a 0,23 , sendo ligeiramente inferiores à média encontrada na literatura (Ribeiro et al., 2001; Biffani et al., 1999). A estimativa de herdabilidade para PE18 foi de 0,39, similar à observada por Gressler (1998), porém mais alta que a citada por Fernandes et al. (1996) e mais baixa que a descrita por Cyrillo et al. (2001). O valor registrado neste estudo pode ser considerado de média à elevada magnitude.

Os valores estimados para efeito do ambiente permanente são semelhantes àqueles relatados na literatura nacional (Souza \& Ramos, 1995; Cardellino, 1987). A herdabilidade estimada para a fertilidade real foi de 0,06 , próximo às estimativas de herdabilidade para características de fertilidade encontradas na literatura (Nájera, 1990; Barbosa,1991). A herdabilidade desta característica apresenta valores entre 0,01 e 0,1 , considerados de baixa magnitude, indicando baixa influência dos genes aditivos sobre a fertilidade e refletindo a necessidade de mudanças na qualidade de alimentação, nos cuidados profiláticosanitários, no manejo reprodutivo, entre outros.

As correlações genéticas (Tabela 4) entre as características de crescimento observadas neste trabalho, referentes às características produtivas entre PD e P18, PD e PE18, e P18 e PE18, foram iguais a
$0,44,0,81$ e 0,46 , respectivamente, sugerindo que a seleção para o aumento de uma acarretará em melhoria da outra, por intermédio de seleção indireta.

Considerando-se o valor de herdabilidade encontrado para IEP $(0,42)$, sugere-se variabilidade genética importante para programa de melhoramento genético, devendo a seleção de animais para esta característica e data do parto ser vista com cuidado (Ponzoni, 1992; McManus \& Hamilton, 1991). A herdabilidade de DP encontrada foi de 0,33 , valor superior ao encontrado por Rege \& Famula (1993), de 0,16. A correlação genética entre IEP e DP foi de $-0,21$ (Tabela 5), como esperarado em rebanhos com esta-

Tabela 4 -Correlações genéticas entre as características de crescimento

Table 4 - Genetic correlations between growth traits

\begin{tabular}{lccc}
\hline Características & P18 & PE18 & PDM \\
Traits & $W 18$ & $S P 18$ & $W W M$ \\
\hline PD $(W W)$ & 0,44 & 0,81 & 0,27 \\
P18 $(W 18)$ & - & 0,46 & 0,68 \\
PE18 $(S P 18)$ & - & - & $-0,12$ \\
\hline
\end{tabular}

* PD - peso à desmama efeito direto; PDM - peso à desmama efeito materno; P18 - peso aos 18 meses de idade; CE18 perímetro escrotal aos 18 meses de idade.

* WW-direct effect for weaning weight; $W W M$ - maternal effect for weaning weight; W18 - weight at 18 months of age; SP18 - scrotal perimeter at 18 months. 
ção de monta definida. Segundo Morris \& Cullen (1988), em rebanhos de estação de monta restrita, a data do parto não seria uma característica importante, em função da correlação negativa com o intervalo de partos. Entretanto, essa correlação negativa significa que as vacas que parem cedo necessitam aguardar um tempo maior para o início da próxima estação de monta que aquelas que parem tardiamente.

A estimativa de herdabilidade para IPP encontrada é de baixa magnitude $(0,05)$, possivelmente em razão da entrada tardia das fêmeas em reprodução, neste caso, aos 42 meses de idade, quando todas as novilhas já tinham atingido a puberdade. Esta estimativa está de acordo com os valores citados em outros trabalhos, podendo variar de 0,01 a 0,25 (Gressler, 1998 e Garnero et al., 1998).

As correlações genéticas para as características reprodutivas encontram-se na Tabela 5. As características avaliadas nas fêmeas apresentaram valores negativos, de magnitude média e alta. No caso da correlação genética da FR com IEP $(-0,90)$, com IPP $(-0,89)$ e com DP $(-0,86)$, obteve-se valor favorável, indicando que, ao se selecionar para aumento da FR (apesar da baixa herdabilidade), os genes envolvidos estarão contribuindo para diminuição dessas outras características.

Para as correlações genéticas entre PE18 do filho e as características reprodutivas da mãe, observaram-se somente estimativas negativas, sendo que a relacionada com DP foi a mais alta $(-0,46)$, corroborando outros relatos (Martins Filho\& Lobo, 1991; Gressler, 1998; Dias et al., 2000). Este resultado significa que seleção no macho para PE18 deve melhorar também características reprodutivas nas fêmeas.

A correlação genética entre IEP e IPP $(-0,69)$ foi discrepante àquela encontrada $(0,47)$ por Nájera (1990). Além disso, os estudos não são coerentes neste assunto, pois, enquanto alguns citam antagonismo entre características produtivas e reprodutivas (Mariante \& Zancaner, 1985; Barbosa, 1991; Mercadante et al., 2000), outros (Mascioli et al., 1999) têm relatado o oposto. As correlações genéticas entre PE18 e as características reprodutivas das fêmeas na população foram negativas, baixas a médias, sendo com DP a mais alta $(-0,46)$.

Bergmann (1999) ressalta que existem evidências na literatura de que a puberdade de machos Nelore, em condições nutricionais adequadas, ocorra próximo ao primeiro ano de idade. Gressler (1998) observou correlação genética favorável entre perímetro escrotal aos 12 meses de idade e data do primeiro parto e desfavorável entre perímetro escrotal aos 18 meses de idade e data do primeiro parto, conjeturando que estes resultados poderiam indicar que a seleção de animais com maior circunferência escrotal aos 12 meses de idade estaria associada à escolha de animais que apresentassem maiores níveis de hormônios gonadotróficos, culminando com a puberdade, o início da atividade reprodutiva de machos e fêmeas e concepções mais precoces na primeira estação de monta, além de antecipação das datas do primeiro parto. Aos 18 meses de idade, a maioria dos machos já estaria em período pós-púbere e a seleção para maiores circunferências escrotais nesta idade estaria associada a maiores pesos corporais e, possivelmente, à menor precocidade reprodutiva em ambientes tropicais. Contudo, ainda são incipientes e contraditórios os estudos sobre perímetro escrotal, que passou a ser avaliado nestes últimos anos, havendo, portanto, a necessidade de mais estudos em ambientes diferentes para se elucidar este relacionamento (Unanian et al., 2000).

\section{Conclusões}

Existem dificuldades para o melhoramento genético sobre a fertilidade em bovinos, principalmente graças à forte influência do meio ambiente sobre estas características. Os resultados obtidos neste trabalho permitem concluir que as características estudadas devem ser corrigidas para os efeitos de meio abordados, por ocasião da avaliação de reprodutores e comparação de animais com vistas à seleção, visando-se a minimização das diferenças resultantes desses efeitos.

$\mathrm{O}$ uso da idade ao primeiro parto e da data do parto como características observadas nas fêmeas, assim como a idade à puberdade avaliada nos machos pelo perímetro escrotal, devem ser incluídas em programas de melhoramento genético na pecuária de corte, sobretudo quando se utiliza estação de monta de curta duração.

De acordo com as estimativas de herdabilidade, concluiu-se que as características estudadas podem responder à seleção, com exceção a FR e IPP. As correlações genéticas entre características produtivas aqui estudadas foram favoráveis, indicando possibilidade da obtenção de progressos genéticos, ou seja, a seleção para o aumento de uma acarretará

R. Bras. Zootec., v.33, n.6, p.1432-1444, 2004 
Tabela 5 - Correlações genéticas entre as características reprodutivas

Table 5 - Genetic correlations among reproductive traits

\begin{tabular}{lcccc}
\hline Característica* & IEP & IPP & DP & PE18 \\
Trait & $C I A$ & $F C C$ & $D S$ & $P 18$ \\
\hline FR $(T F)$ & $-0,90$ & $-0,89$ & $-0,86$ & $-0,12$ \\
IDP $(C I)$ & - & $-0,69$ & $-0,21$ & $-0,19$ \\
IPP $(A F C)$ & - & - & $-0,30$ & $-0,23$ \\
DP $(C D)$ & - & - & - & $-0,46$
\end{tabular}

${ }^{\star}$ Fertilidade real (FR), intervalo de partos (IEP), idade ao primeiro parto (IPP), dias do parto (DP) e perímetro escrotal aos 18 meses de idade (PE18).

Scrotal perimeter at 18 months (SP18), calving interval (Cl), age at first calving $(A F C)$, calving date $(C D)$ and true fertility (TF).

melhoria da outra. As correlações entre características reprodutivas das fêmeas foram, em geral, favoráveis, o mesmo ocorrendo entre elas e PE18 nos machos.

Ressalta-se que os resultados nesta pesquisa referem-se especificamente ao rebanho analisado e às condições ambientais da região, merecendo cautela à aplicação prática destas conclusões para uso generalizado.

\section{Literatura Citada}

ARIAS, A.; SLOBODZIAN, A. Análisis de los datos de los registros de la raza Nelore de Paraguay. In: CONGRESSO INTERNACIONAL DE PRODUCCIÓN DE CARNE, 1998, Anales... Asunción: APCN/BURSAL/DBO Sul. 1998. p.51-94.

BARBOSA, P.F. Análise genético-quantitativa de características de crescimento e reprodutivas em fêmeas da raça Canchim. Ribeirão Preto: Universidade de São Paulo, 1991. 96p. Tese (Doutorado em Genética) - Universidade de São Paulo, 1991.

BERGMANN, J.A.G. Seleção de zebuínos para precocidade sexual. In: SIMPÓSIO DE PRODUÇÃO DE GADO DE CORTE, 1999, Belo Horizonte. Anais... Belo Horizonte: Universidade Federal de Minas Gerais, 1999.

BERGMANN, J.A.G. Melhoramento genético da eficiência reprodutiva em bovinos de corte. In: CONGRESSO BRASILEIRO DE REPRODUÇÃO ANIMAL, 1993, Belo Horizonte. Anais... Belo Horizonte: Colégio Brasileiro de Reproduçãp Animal, 1993. p.70-86. (Suplemento)

BERGMANN, J.A.G.; ZAMBORLINI, L.C.; PROCOPIO, C.S.O. et al. Estimativas de parâmetros genéticos do perímetro escrotal e do peso corporal em animais da raça Nelore. Arquivo Brasileiro de Médicina Veterinária e Zootecnia, v.48, p.69-78, 1996.

BIFFANI, S.; MARTINS FILHO, R.; MARTINI, A. et al. Fatores ambientais e genéticos sobre o crescimento ao ano e ao sobreano de bovinos Nelore, criados no Nordeste do
Brasil. Revista Brasileira de Zootecnia, v.28, n.3, p.468472, 1999.

BOLDMAN, K.G.; KRIESE, L.A.; Van VLECK, L.D. et al. A manual for use of MTDFREML: a set of programs to obtain estimates of variance and (co)variance (DRAFT). Lincoln: Department of Agriculture/ARS, 1995. 120p.

BOURDON, R.M.; BRINKS, J.S. Calving date versus calving interval as a reproductive measure in beef cattle. Journal of Animal Science, v.57, p.1412-1417, 1983.

CAMPELLO, C.C.; MARTINS FILHO, R.; LOBO, R.N.B. Intervalo de partos e fertilidade real em vacas nelore no estado do Maranhão. Revista Brasileira de Zootecnia, v.28, n.3, p.474-479, 1999.

CARDELLINO, R.A. Repetibilidade do peso ao nascer, peso à desmama e ganho de peso pré-desmama em bovinos da raça Nelore. In: REUNIÃO ANUAL DA SOCIEDADE BRASILEIRA DE ZOOTECNIA, 24., 1987, Brasília. Anais... Brasília: Sociedade Brasileira de Zootecnia, 1987. p.277.

CARDELliNO, R.A.; PONS, S.B. Parâmetros genéticos do intervalo entre partos em bovinos da raça nelore. Pesquisa Agropecuária Brasileira, v.22, n.3, p.305-310, 1987.

CYRILLO, J.N.S.G.; RAZOOK, A.G.; FIGUEIREDO, L.A. et al. Estimativas de tendências e parâmetros genéticos do peso padronizado aos 378 dias de idade, medidas corporais e perímetro escrotal de machos Nelore de Sertãozinho, SP. Revista Brasileira de Zootecnia, v.30, n.1, p.56-65, 2001.

DIAS, L.T.; FARO, L.E.; FRIES, L.A. et al. Estimativas de parâmetros genéticos para perímetro escrotal e idade ao primeiro parto em animais da raça Nelore. In: REUNIÃO ANUAL DA SOCIEDADE BRASILEIRA DE ZOOTECNIA, 37., 2000, Viçosa, MG. Anais... Viçosa, MG: Sociedade Brasileira de Zootecnia, 2000. p.223.

ELER, J.P.; FERRAZ, J.B.S.; LOBO, R.B. et al. Genetic antagonism between growth and maternal ability in Nellore cattle. Revista Brasileira de Genética, v.17, p.59-64, 1994.

ELER, J.P.; Van VLECK, L.D.; FERRAZ, J.B.S. et al. Estimation of variances due to direct and maternal effects for growth traits of Nellore cattle. Journal of Animal Science, v.73, n.4, p.3253-3258, 1995.

FERNANDES, A.; MAGNABOSCO, C.U.; OJALA, M. et al. Estimativas de parâmetros genéticos e ambientais de medidas corporais e peso de bovinos a raça Brahman nos trópicos. In: REUNIÃO ANUAL DA SOCIEDADE BRASILEIRA DE ZOOTECNIA, 33., 1996, Fortaleza. Anais... Fortaleza: Sociedade Brasileira de Zootecnia, 1996. p.65-67.

GARNERO, A.V.; LÔBO, R.B.; BORJAS, A.R. et al. Estimativas de parâmetros genéticos para características incluídas em critérios de seleção em gado de corte. In REUNIÃO ANUAL DA SOCIEDADE BRASILEIRA DE ZOOTECNIA, 35., 1998, Botucatu. Anais... Botucatu: Sociedade Brasileira de Zootecnia, 1998. p.434-436.

GIANNONI, M.A.; GIANNONI, M.L. Genética e melhoramento de rebanho nos trópicos. Jaboticabal: Universidade Etadual de São Paulo, 1989. 687p.

GRESSLER, S.L. Estudo de fatores de ambiente e parâmetros genéticos de algumas características reprodutivas em animais da raça Nelore. Belo Horizonte: Universidade Federal de Minas Gerais, 1998. 72p. Dissertação (Mestrado em Zootecnia) - Universidade Federal de Minas Gerais, 1998.

HANSEN, L.B.; FREEMAN, A.E.; BERGER, P.J. Yield and fertility relationship in dairy cattle. Journal of Dairy Science, v.66, n.1, p.293-305, 1983. 
MAGNABOSCO, C.U.; FAMULA, T.R.; LÔBO, R.B. et al. Estimativas de parâmetros genéticos e ambiente de características de crescimento em bovinos da raça Nelore. In: REUNIÃO ANUAL DA SOCIEDADE BRASILEIRA DE ZOOTECNIA, 33., 1996, Fortaleza. Anais... Fortaleza, 1996, p.142-144.

MARIANTE, A.S.; ZANCANER, A. Crescimento e reprodução em gado Nelore: visão do criador e do pesquisador. São Paulo: Criadores, 1985. 152p.

MARTINS FILHO, R.; LÔBO, R.B. Estimates of genetic correlations between sire scrotal circumference and offspring age at first calving in Nellore cattle. Revista Brasileira de Genética, v.14, n.1, p.209-212, 1991.

MASCIOLI, A.S.; ALENCAR, M.M.; BARBOSA, P.F. Influências de fatores de meio sobre pesos de animais da raça Canchim. Revista Brasileira de Zootecnia, v.25, n.1, p.72-82, 1996.

MASCIOLI, A.S.; ALENCAR, M.M.; TALHARI, F.M. et al. Correlações genéticas entre peso aos 12 meses de idade e características reprodutivas e de crescimento em fêmeas, na raça Canchim In: REUNIÃO ANUAL DA SOCIEDADE BRASILEIRA DE ZOOTECNIA, 36. ,1999, Porto Alegre, Anais... Porto Alegre: Sociedade Brasileira de Zootecnia, 1999. 3p.

McMANUS, C.; HAMILTON, W. Estimation of genetic and phenotypic parameters for growth and reproductive traits for red deer on an upland farm. Animal Production, n.53, n.1, p.227-235, 1991.

McMANUS, C.M.; SAUERESSIG, M. G.; FALÇÃO, R. et al. Componentes reprodutivos e produtivos no rebanho mestiço de corte da Embrapa Cerrados. Revista Brasileira de Zootecnia, v.31, n.2, p.648-657, 2002.

MERCADANTE, M.E.; LÔBO, R. Estimativas de (co) variâncias e parâmetros genéticos dos efeitos diretos e materno de características de crescimento de fêmeas de um rebanho Nelore. Revista Brasileira de Zootecnia, v.26, n.6, p.1124-1133, 1997.

MERCADANTE, M.E.; LÔBO, R.; OLIVEIRA, H.N. Estimativas de (co)variâncias entre características de reprodução e de crescimento em fêmeas de um rebanho Nelore. Revista Brasileira de Zootecnia, v.29, n.4, p.997-1004, 2000.

MILAGRES, J.C.; SILVA, L.O.C.; NOBRE, P.R.C. Influência de fatores de meio e herança sobre pesos de animais da raça Nelore no Estado de Minas Gerais. Revista da Sociedade Brasileira de Zootecnia, v.14, n.4, p.463-484, 1985.

MIRANDA, J.J.F.; CARNEIRO, G.G.; PENNA, V.M. et al. Estudo dos fatores ambientais relacionados com a idade à primeira cria na raça Nelore. Arquivos Brasileiros de Medicina Veterinária e Zootecnia, v.34, n.2, p.375-380, 1982.

MORRIS C.A.; CULLEN N.G. Estrous and reproductive performance of early calving and late calving beef cows. New Zealand Journal of Agricultural Research, v.31, n.1, p.395-399, 1988.

NÁJERA, A.J.M. Efeitos genéticos e não genéticos sobre características reprodutivas e ponderais de duas populações de bovinos da raça Nelore. Belo Horizonte: Universidade Federal de Minas Gerais, 1990. 150p. Dissertação (Mestrado em Melhoramento Animal) - Universidade Federal de Minas Gerais, 1990.

NÁJERA, A.J.M.; OLIVEIRA, H.N.; PEREIRA, J.C.C. Efeitos genéticos e não genéticos sobre características ponderais de duas populações de bovinos da raça nelore. Arquivos Brasileiros de Medicina Veterinária e Zootecnia, v.43, n.1, p.81-92, 1991.
NEELY, J.D.; JOHNSON, B.H.; DILLARD, E.U. et al. Genetic parameters for testes size and sperm number in Hereford bulls. Journal of Animal Science, v.55, n.5, p.1033-1039, 1982.

NOBRE, P.R.C.; ROSA, A.N.; SILVA, L.O.C. Influência de fatores genéticos e de meio sobre os pesos de gado Nelore no Estado da Bahia. Revista Brasileira de Zootecnia, v.14, n.3, p.338-357, 1985.

NOTTER, D.R. Maximizing fertility in animal breeding programs. Blacksburg : Virginia Polytechnic Institute State University, Department of Animal and Poultry Sciences, 1995. p. in reg. (XI Congresso Brasileiro de Reprodução Animal. Belo Horizonte, 1995, pré- congresso).

OLIVEIRA, J.A. Estudo genético quantitativo de desenvolvimento ponderal do gado Canchim. Ribeirão Preto: Universidade de São Paulo, 1979, 87p. Dissertação (Mestrado em Genética) - Universidade Federal de Minas Gerais, 1979.

OLIVEIRA, J.A.L.; ALENCAR, M.M.; LIMA, R. Eficiência produtiva de vacas da raça Nelore. Revista Brasileira de Zootecnia, v.24, n.3, p.445-452, 1995.

PÄDUA, J.T.; MUNARI, D.P.; WATANABE, Y.F. et al. Avaliação de efeitos de ambiente e da repetibilidade de características reprodutivas em bovinos da raça Nelore. Revista Brasileira de Zootecnia, v.23, n. 1, p.126-139, 1994.

PEREIRA, J.C.C. Melhoramento genético aplicado à produção animal. Belo Horizonte: Universidade Federal de Minas Gerais, 1999. 416p.

PEROTTO, D.O. Efeito materno no melhoramento de gado de corte. In: Curso de Melhoramento de Gado de Corte da EMBRAPA - GENEPLUS, 4., Campo Grande, 2000.

PONZONI, R.W. Which trait for genetic improvement of beef cattle reproduction: calving rate or calving day. Journal of Animal Breed Genetics, v.10, p.119-128, 1992.

REGE, J.E.O; FAMULA, T.R. Factors affecting calving date and its relationship with production traits of Hereford dams. Animal Production, v.57, n.1, p.385-395, 1993.

RIBEIRO, M.N.; PIMENTA FILHO, E.C.P.; MARTINS, G.A. et al. Herdabilidade para efeitos direto e materno de características de crescimento de bovinos Nelore no estado de Paraíba. Revista Brasileira de Zootecnia, v.30, n.4, p.1224-1227, 2001.

ROSA, A.N.; LÔBO R.B. Considerações sobre o tamanho adulto de matrizes Nelore. In: SIMPÓSIO NACIONAL DA SOCIEDADE BRASILEIRA DE MELHORAMENTO ANIMAL, 2., 1998, Uberaba. Anais... Uberaba: Sociedade Brasileira de Melhoramento Animal, 1998.

ROSA, A.N. In: Curso de Melhoramento de Gado de Corte da EMBRAPA - GENEPLUS, 5., Campo Grande, 2001.

ROSA, A.N., LÔBO R.B., OLIVEIRA, H.N. et al. Peso adulto de matrizes em rebanhos de seleção da raça Nelore no Brasil. Revista Brasileira de Zootecnia, v.30, n.3, p.1027-1036, 2001, Suplemento 1.

STATISTICAL ANALYSIS SYSTEM - SAS. User's Guide, Version 8, Cary: 1999. 295p.

SILVA, J.A.V.; RAZOOK, A.G.; TONHATI, H. et al. Effects of selection for post weaning weight on Nellore cow productivity efficiency indicators. Revista Brasileira de Zootecnia, v.29, n.4, p.1020-1027, 2000.

SIQUEIRA, R.L.P.G.; OLIVEIRA, J.A.; LÔBO, R.B. et al. Análise da variabilidade genética aditiva de características de crescimento na raça Nelore. Revista Brasileira de Zootecnia, v.32, n.1, p.99-105, 2003.

SMITH, B.A.; BRINKS, J.S.; RICHARDSON, G.V. Estimation of genetic parameters among reproductive and growth traits

R. Bras. Zootec., v.33, n.6, p.1432-1444, 2004 
1444 Fatores Ambientais e Parâmetros Genéticos para Características Produtivas e Reprodutivas em um Rebanho...

in yearling heifers. Journal of Animal Science, v. 67, n.11, p.2886-2891, 1989.

SOUZA, J.C.; FERRAZ FILHO, P.B.; VALENCIA, E.F.T. et al. Estudo comparativo de peso ao desmame de bezerros filhos de touros Zebu e Europeu. In: REUNIÃO ANUAL DA SOCIEDADE BRASILEIRA DE ZOOTECNIA, 31., 1994, Maringá. Anais... Maringá: Sociedade Brasileira de Zootecnia, 1994. p.181.

SOUZA, J.C.; RAMOS, A.A. Efeitos de fatores genéticos e do meio sobre pesos de bovinos a raça Nelore. Revista Brasileira de Zootecnia, v.24, n.1, p.164-172, 1995.

TEIXEIRA, R.A. Comparações bio-econômicas entre dois sistemas de produção com diferentes níveis de fertilidade em rebanho Nelore a pasto. Jaboticabal: Universidade Estadual Paulista , 1997. Monografia (Graduação) - Universidade Estadual Paulista, 1997.
TROVO, J.B.F.; RAZOOK, A.G. I Curso sobre Avaliação Genética de Bovinos de Corte em Goiás. PMGRN/ USP Ribeirão Preto, setembro/1996.

UNANIAN, M.M.R.V.; SILVA, A.E.D.F.; McMANUS, C.M. et al. Características biométricas testiculares para avaliação de touros zebuínos da raça Nelore. Revista Brasileira de Zootecnia, v.29, n.1, p.177-204, 2000.

Recebido em: 17/11/02

Aceito em: 22/04/04 